\title{
Design nanocrack patterns in heterogeneous films
}

\author{
David Salac and Wei $\mathrm{Lu}^{1}$ \\ Department of Mechanical Engineering, University of Michigan, Ann Arbor, MI 48109, USA \\ E-mail: weilu@umich.edu
}

Received 23 May 2006, in final form 5 September 2006

Published 28 September 2006

Online at stacks.iop.org/Nano/17/5185

\begin{abstract}
Nanowires have significant potential in future technologies such as nanomechanical devices and electronics. Recent experiments suggest that nanowires with sub-100 nm diameters may be fabricated by filling cracks with various materials. The geometry of cracks becomes important on such a length scale, and the practical application of the approach requires an understanding of crack evolution in heterogeneous films. This paper proposes a level-set approach to model directed nanocracks on pre-patterned substrates. The approach does not require the explicit tracking of crack fronts and thus allows the simulation of complex crack patterns. Results indicate that pre-patterning a substrate can lead to various well controlled nanocrack patterns, suggesting a possibility to make designed and complex nanowires difficult to obtain with other methods.
\end{abstract}

\section{Introduction}

Thin film cracking has been observed experimentally in different materials systems [1, 2] and length scales [3, 4]. While cracking of material is generally considered undesirable, recent study suggests that the mechanism may be used for nanowire fabrication $[5,6]$. Nanowires are typically formed of semi-conductor or metallic materials, and can have diameters of $10-100 \mathrm{~nm}$ and lengths of up to $10 \mu \mathrm{m}$. They have received significant interest as functional components for nanoscale systems [7, 8]. Typically, nanowires have been fabricated by techniques such as scanning tunnelling microscopy $[9,10]$ and electrochemical deposition [11-13]. Controlled formation of nanocrack patterns suggests an efficient low-cost method that combines the top-down and bottom-up approaches [5]. In the process a $\mathrm{SiO}_{2}$ film was patterned by etching sharp corners for crack initiation. Elevating the system temperature induces tensile stress in the film, which causes cracks to form in predetermined directions. These cracks are then filled with a suitable material, such as nickel, and the subsequent removal of $\mathrm{SiO}_{2}$ produces a controlled network of nanowires of about $100 \mathrm{~nm}$ in diameter.

While the experimental work highlights the potential of using crack patterns to form nanowires, only simple crack propagation directions have been applied, such as straight lines

1 Author to whom any correspondence should be addressed. and gentle curves. A computational model to predict complex crack paths in heterogeneous films would be necessary to fully explore the potential, which allows the development of novel nanowire paths and nanocircuits. Numerical methods such as finite element analysis [14] and the cohesive zone model [15] have been widely used to study crack propagation. These approaches require tracking the crack path and front explicitly. Thus the algorithm and computation become very complicated for multiple cracks, non-straight cracks, or crack intersections. Resolving nanoscale features requires consideration of a finite crack tip radius. The assumption about the shape and extension of the crack tip used in the finite element analysis or cohesive zone model may not be applicable at the small length scales needed for fracturebased nanowire fabrication. Recently, phase field models have been extended to study crack problems, where the crack surface is a transition region described by a continuous field variable [16-20]. The approach eliminates the need to explicitly describe the location of cracks [21], which allows the investigation of a large numbers of cracks without complex meshes. However, there is mathematical ambiguity about the actual location of crack boundaries and the definition of interface properties in a phase field model. The scale of the resulting crack patterns can be blurred or overwhelmed by the width of the phase field interface. This approach also introduces several user-controlled parameters that are hard to directly relate to physically measurable quantities. 


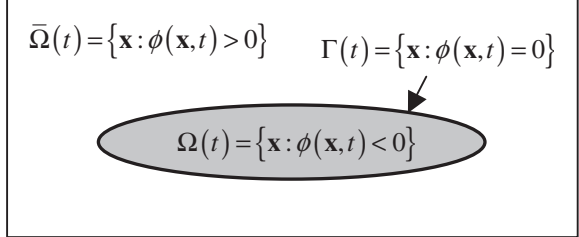

Figure 1. A schematic diagram of the level-set regions. The zero contour of the level set corresponds to the interface $\Gamma(t)$, which separates the domain into two regions: $\phi(\mathbf{x}, t)<0$ in $\Omega(t)$ and $\phi(\mathbf{x}, t)>0$ in $\Omega(t)$.

In this paper we propose a level-set approach to investigate the formation of nanoscale crack patterns. Compared to the phase field approach that we and other researches have used for crack problems, the present work allows more physically realistic description of nanocracks. In reality, crack interfaces are physically sharp on the atomic scale. Capturing the geometry would be significant for applications such as nanowires, where the phase field approach cannot work well due to the ambiguity about the actual location of crack boundaries and the definition of interface properties. The proposed level-set approach allows simulation of many cracks and provides the precise location of crack interfaces, while it easily accommodates large morphological changes without the need of expensive re-meshing. In addition, the proposed model can handle cracking physics more naturally than previous methods, which need special considerations at the crack tips. In contrast to other models, this model uses a minimum number of phenomenological parameters. The application of the level-set approach in other problems has already demonstrated its efficiency and capability to handle complex and large morphological changes such as pinching and combining [22-24]. While the level-set approach has also been explored to investigate the cracking of materials [25], these studies are limited to the propagation of a single crack and require finite element analysis to obtain the elastic field in the system. In contrast, this paper investigates the propagation of many cracks and how pre-patterns and elastic interactions affect the crack morphology. An iterative method is developed to obtain the elastic field, which results in a scheme that is both fast and easy to implement. The plan of this paper is as the following. Section 2 introduces the levelset approach. The model for crack patterns is formulated in section 3. Simulations relevant to nanocrack patterns and nanowire structures are presented in section 4 . Section 5 is the conclusion.

\section{The level-set approach}

First introduced by Sethian and Osher [26], the levelset approach has proven very powerful in describing a wide variety of interface evolution problems, including evolving fluid interfaces [27], epitaxial growth [28] and electromigration [29]. The level-set approach works by embedding an interface into a function of higher dimensionality. To explain the concept, consider a timedependent closed interface $\Gamma(t)$, as shown in figure 1. Denote the enclosed region by $\Omega(t)$. The region outside is given by

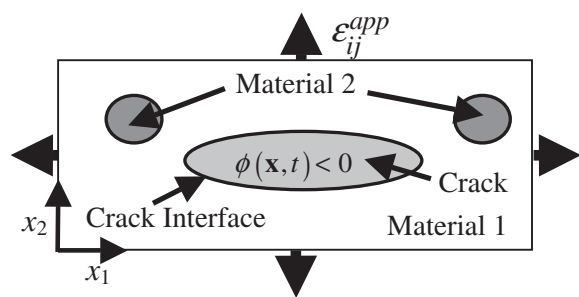

Figure 2. A heterogeneous film containing a crack. The crack region is represented by $\phi(\mathbf{x}, t)<0$.

$\bar{\Omega}(t)$. Define a spatially continuous, arbitrary function $\phi(\mathbf{x}, t)$ such that

$$
\begin{array}{ll}
\phi(\mathbf{x}, t)<0 & \text { in } \Omega(t) \\
\phi(\mathbf{x}, t)=0 & \text { on } \Gamma(t) \\
\phi(\mathbf{x}, t)>0 & \text { in } \bar{\Omega}(t) .
\end{array}
$$

Here $\mathbf{x}$ denotes the position vector. A level-set function can be any function that satisfies the requirement of equation (1). Thus for any known interface a level set function can be constructed. Conversely, the zero contour of the level-set function determines the location of the interface. Specifically, as discussed in section 3.3, this paper uses the signed distance function for the level set. The normal of the interface, $\mathbf{n}$, and the curvature, $\kappa$, are expressed by [23]

$$
\begin{gathered}
\mathbf{n}=\frac{\nabla \phi}{|\nabla \phi|}, \\
\kappa=\nabla \cdot \frac{\nabla \phi}{|\nabla \phi|} .
\end{gathered}
$$

The positive normal direction points out from $\Omega(t)$, i.e. from the region of negative $\phi(\mathbf{x}, t)$ to the positive region. The curvature is positive for a convex surface of $\Omega(t)$.

The zero contour of the level-set function moves along with the interface. The motion of the level set function is described by convecting $\phi(\mathbf{x}, t)$, namely

$$
\frac{\partial \phi}{\partial t}+\mathbf{v} \cdot \nabla \phi=0
$$

where $\mathbf{v}$ is the interface velocity on the interface and can have arbitrary values elsewhere. Movement tangential to the interface does not cause any configurational changes. Thus it is only necessary to consider the normal velocity of the interface $v_{\mathrm{n}}$. Projecting the velocity in the normal direction $\mathbf{n}$ gives the standard level-set evolution equation [23],

$$
\frac{\partial \phi}{\partial t}+v_{\mathrm{n}}|\nabla \phi|=0
$$

Thus, calculating the normal velocity and advancing the level-set function by equation (5) determines the interface movement.

\section{A level-set model for crack patterns}

Consider a heterogeneous film shown in figure 2, which comprises two materials and a crack. Define a level-set function $\phi(\mathbf{x}, t)$ such that a crack takes $\phi(\mathbf{x}, t)<0$ while the 
Design nanocrack patterns in heterogeneous films

crack surface is given by $\phi(\mathbf{x}, t)=0$. The excess chemical potential at the crack surface relative to that of a material point inside a reference unstressed film is

$$
\Delta \mu=\Omega\left(g_{\mathrm{e}}-\gamma \kappa\right) .
$$

Here $\Omega$ is the atomic volume, $g_{\mathrm{e}}$ is the elastic energy density and $\gamma$ is the interfacial energy per unit area. This paper considers quasi-static crack growth, assuming that the elastic field in the system equilibrates at a much faster rate than the crack propagation. The fracture dynamics is not considered. The normal velocity relates to the excess chemical potential by $v_{\mathrm{n}}=D \Delta \mu / \gamma \Omega$, where $D$ is a phenomenological kinetic coefficient [20]. The kinetics is analogous to the time dependent Ginzburg-Landau equation, where it acts to reduce the free energy of a material system. This paper focuses on the direction and path of the crack propagation. In this sense, $D$ can be viewed as an adjustable numerical parameter. A larger $D$ allows a crack to extend faster. This model does not predict crack velocity. The crack velocity from simulations can be made to match experimental results by choosing an appropriate value for $D[30,31]$. Note that the crack direction is not determined by a global rule, but by the collective behaviours of all the points close to the crack surface. In each time step the movement of all the points close to a crack surface forms a new crack profile. Thus a crack not only changes its direction, but also its shape. A crack, once formed, often stays in the material even after the load is removed. To model the irreversibility of the cracking process we restrict ourselves to positive normal velocity, namely

$$
v_{\mathrm{n}}=\max \left(\frac{D}{\gamma \Omega} \Delta \mu, 0\right) .
$$

The curvature $\kappa$ can be computed easily using equation (3). The following discusses the calculation of the elastic energy density $g_{\mathrm{e}}$

\subsection{The elastic field}

Elastic stiffness at a spatial point can be obtained by interpolation [32], which gives

$$
\lambda_{i j l m}(\mathbf{x})=\rho(\mathbf{x}) \lambda_{i j l m}^{\mathrm{f}} .
$$

Here $\lambda_{i j l m}^{\mathrm{f}}(\mathbf{x})$ is the film stiffness and $\rho(\mathbf{x})$ is a density function, which smoothly transitions from a value of zero inside the crack to a value of unity outside the crack. A continuous $\rho(\mathbf{x})$ is for computational convenience. It should be noted that the choice of $\rho(\mathbf{x})$ for the elastic calculation is independent of the level-set equations. Thus the resolution of the crack geometry and that of the elastic field is completely separated. This level-set approach allows resolving sharp crack interfaces and simultaneously offers the flexibility of numerical control in the calculation of elasticity, which is in contact to the diffuse interface model [19]. The specific form of $\rho(\mathbf{x})$ is insignificant as long as the transition is narrow. Our simulations have shown that while the magnitude of the elastic field around the crack tip may be affected, the crack direction is insensitive to $\rho(\mathbf{x})$. A convenient choice is

$$
\rho(\mathbf{x})=\frac{1}{2}\left[1+\tanh \left(\frac{2 \phi(\mathbf{x})}{h}\right)\right],
$$

where $h$ is a parameter controlling the transition thickness.
Denote the lattice misfit between the film material and the substrate by $\varepsilon_{0} \delta_{i j}$, where $\delta_{i j}$ is the Kronecker delta. The misfit strain at any spatial point in a crack-containing film is given by

$$
\varepsilon_{i j}^{0}(\mathbf{x})=(1-\rho(\mathbf{x})) \varepsilon_{0} \delta_{i j} .
$$

The elastic field can be obtained by the supposition of a uniform field and an inhomogeneous perturbation field. The total strain field, $\varepsilon_{i j}(\mathbf{x})$, is expressed by

$$
\varepsilon_{i j}(\mathbf{x})=\bar{\varepsilon}_{i j}+\tilde{\varepsilon}_{i j}(\mathbf{x}),
$$

where $\bar{\varepsilon}_{i j}$ is the uniform strain and $\tilde{\varepsilon}_{i j}(\mathbf{x})$ is the inhomogeneous strain. The homogeneous strain is the uniform macroscopic strain characterizing the macroscopic shape and volume change associated with the total strain, $\varepsilon_{i j}(\mathbf{x})$. It relates to the macroscopic applied stress by the volume average of the system stiffness. The inhomogeneous strain, $\tilde{\varepsilon}_{i j}(\mathbf{x})$, relates to the perturbation displacement, $u_{i}(\mathbf{x})$, by

$$
\tilde{\varepsilon}_{i j}(\mathbf{x})=\frac{1}{2}\left(\frac{\partial u_{i}(\mathbf{x})}{\partial x_{j}}+\frac{\partial u_{j}(\mathbf{x})}{\partial x_{i}}\right) .
$$

The total stress field, $\sigma_{i j}(\mathbf{x})$, can be computed by

$$
\sigma_{i j}(\mathbf{x})=\lambda_{i j l m}(\mathbf{x})\left(\varepsilon_{l m}(\mathbf{x})-\varepsilon_{l m}^{0}(\mathbf{x})\right) .
$$

In most situations the elastic field reaches equilibrium much faster than the crack propagation. The stress field satisfies the standard elastic equation,

$$
\frac{\partial \sigma_{i j}(\mathbf{x})}{\partial x_{j}}=0
$$

Substitute equations (11)-(13) in equation (14), we obtain a partial differential equation for the displacement field,

$$
\frac{\partial}{\partial x_{j}}\left[\lambda_{i j l m}(\mathbf{x}) \frac{\partial u_{l}(\mathbf{x})}{\partial x_{m}}\right]=\frac{\partial}{\partial x_{j}}\left[\lambda_{i j l m}(\mathbf{x})\left(\varepsilon_{l m}^{0}(\mathbf{x})-\bar{\varepsilon}_{l m}\right)\right] .
$$

To ensure numerical stability, we add a term of $A_{i j l m} \partial^{2} u_{l} / \partial x_{j} \partial$ $x_{m}$ to both sides of equation (15), where $A_{i j l m}$ is a chosen constant for computational stability. Rearranging the terms gives

$$
\begin{gathered}
A_{i j l m} \frac{\partial^{2} u_{l}(\mathbf{x})}{\partial x_{j} \partial x_{m}}=\frac{\partial}{\partial x_{j}}\left[\lambda_{i j l m}(\mathbf{x})\left(\varepsilon_{l m}^{0}(\mathbf{x})-\bar{\varepsilon}_{l m}\right)\right] \\
-\frac{\partial}{\partial x_{j}}\left[\left(\lambda_{i j l m}(\mathbf{x})-A_{i j l m}\right) \frac{\partial u_{l}(\mathbf{x})}{\partial x_{m}}\right] .
\end{gathered}
$$

Take the Fourier transform of equation (16) and solve the equation by iteration. The displacement field at the $n$th iteration is given by

$$
\begin{gathered}
\hat{u}_{p}^{(n)}(\mathbf{k})=-\sqrt{-1} k_{j} G_{i p}(\mathbf{k})\left[\lambda_{i j l m}(\mathbf{x})\left(\varepsilon_{l m}^{0}(\mathbf{x})-\bar{\varepsilon}_{l m}\right)\right. \\
\left.-\left(\lambda_{i j l m}(\mathbf{x})-A_{i j l m}\right) \frac{\partial u_{l}^{(n-1)}(\mathbf{x})}{\partial x_{m}}\right]_{k},
\end{gathered}
$$

where $\mathbf{k}$ is the wavevector in Fourier space and $G_{i p}(\mathbf{k})=$ $\left(A_{i j p m} k_{j} k_{m}\right)^{-1}$ is the Green's tensor. The hat or a subscript ' $k$ ' denotes the Fourier transform. The initial value is taken to be $\hat{u}_{p}^{(0)}(\mathbf{k})=-\mathrm{i} k_{j} G_{i p}(\mathbf{k})\left[\lambda_{i j l m}\left(\varepsilon_{l m}^{0}-\bar{\varepsilon}_{l m}\right)\right]_{\mathbf{k}}$. The gradient, $\partial u_{l}^{(n-1)}(\mathbf{x}) / \partial x_{m}$, is obtained by inverse Fourier transform of 
$\sqrt{-1} k_{m} \hat{u}_{l}^{(n-1)}(\mathbf{k})$. The convergence of equation (17) depends on the choice of $A_{i j l m}$. We chose $A_{i j l m}=\lambda_{i j l m}^{\mathrm{f}}$, and found that equation (17) converged after about ten iterations. In the situation where the film comprises multiple materials, we may choose $A_{i j l m}$ to be the elastic tensor of the stiffest material. After the displacement field is determined, the elastic energy density is given by

$$
g_{\mathrm{e}}=\frac{1}{2} \lambda_{i j l m}\left(\varepsilon_{i j}-\varepsilon_{i j}^{0}\right)\left(\varepsilon_{l m}-\varepsilon_{l m}^{0}\right) .
$$

\subsection{Level-set evolution}

The propagation of cracks is captured by the evolution of the level set. Choose a characteristic length scale, $l_{0}$, which reflects the feature size to be resolved. Define a characteristic energy density by $\omega_{0}=\gamma / l_{0}$. A characteristic time is defined by $t_{0}=l_{0}^{2} / D$. From a computational point of view, an appropriate $l_{0}$ allows resolution of the physics relevant to the size scale without dealing with extremely large or small numbers since they may cause important information to be lost in numerical noise. Taking $l_{0} \sim 1 \mathrm{~nm}$ allows us to zoom in to reveal nanoscale features. Equation (5) retains its form after normalization, except that now $t$ stands for the normalized time $t / t_{0}$, the gradient operates on the normalized space $\mathbf{x} / l_{0}$, and $v_{\mathrm{n}}=\max \left(g_{\mathrm{e}}-\kappa, 0\right)$ stands for the normalized normal velocity. Here $g_{\mathrm{e}}$ is the elastic energy density normalized by $\omega_{0}$ and $\kappa$ is the curvature normalized by $l_{0}$.

Essentially non-oscillatory and weighted essentially non-oscillatory type schemes have been used to solve equation (5) [23]. These schemes are based on explicit methods, and thus require relatively small time steps. Here we propose a semi-implicit method which has strong numerical stability and allows the usage of large time steps [33]. Rewrite the normalized equation (5) by

$$
\frac{\partial \phi}{\partial t}-\beta \nabla^{2} \phi=S(\phi)-\beta \nabla^{2} \phi,
$$

where $\beta$ is a stabilization constant and $S(\phi)=-v_{\mathrm{n}}|\nabla \phi|$. The idea is to treat the linear term $\beta \nabla^{2} \phi$ on the left implicitly and treat the $S(\phi)-\beta \nabla^{2} \phi$ term on the right explicitly. Replacing $\partial \phi / \partial t$ by $\left(\phi^{(n)}-\phi^{(n-1)}\right) / \Delta t, \beta \nabla^{2} \phi$ by $\beta \nabla^{2} \phi^{(n)}$, and $S(\phi)-$ $\beta \nabla^{2} \phi$ by $S\left(\phi^{(n-1)}\right)-\beta \nabla^{2} \phi^{(n-1)}$ in equation (19) and applying the Fourier transform, we obtain

$$
\hat{\phi}^{(n)}(\mathbf{k})=\hat{\phi}^{(n-1)}(\mathbf{k})+\frac{\Delta t}{1+\beta \Delta t k^{2}}\left[S\left(\phi^{(n-1)}(\mathbf{x})\right)\right]_{\mathbf{k}} .
$$

Here $k^{2}=k_{1}^{2}+k_{2}^{2}$ with $k_{1}$ and $k_{2}$ being the components of the wavevector, and $\Delta t$ is the time step. This semi-implicit approach can significantly alleviate the time step constraint. We have obtained numerical stability in all our simulations by taking $\beta=0.5$.

Without explicit interface tracking, the level-set approach applies equation (20) to evolve $\phi$ in the entire domain. However, $S$ is only defined at the interface. The expression in the non-interface regions needs to be determined. In practice, we first calculate $S$ at the interface, and then use it as the boundary condition to construct the $S$ field in other regions by the following hyperbolic equation [23]:

$$
\frac{\partial S}{\partial \tau}+\operatorname{sgn}(\phi) \frac{\nabla \phi}{|\nabla \phi|} \cdot \nabla S=0 .
$$

Here $\tau$ is time and $\operatorname{sgn}(\phi)$ is the standard sgn function. We write in discrete form and compute equation (21) with an upwind scheme until it reaches a steady state. A similar approach has also been used by other researchers [23].

\subsection{The level-set function}

Equation (5) suggests that the rate at which the level set advances directly relates to its gradient at any spatial point. A well behaved level-set function requires the gradient to be bounded. A widely used method is to set the level set to a signed distance function such that the gradient is unity, $|\nabla \phi|=$ $1[34,35]$. In most implementations the bounded gradient is obtained by regularly replacing the current level set with a signed distance function that has the same zero contour. This process is known as reinitialization. We achieve reinitialization by using the information propagating off the interface. The concept is the following. Imagine a particle moving normal to the interface with a constant speed of unity. The amount of time that the particle has been travelling is equal to its distance from the closest interface point. Mathematically this can be modelled by solving a differential equation in the form of

$$
\frac{\partial \phi}{\partial \tau}+\operatorname{sgn}\left(\phi^{0}\right) \frac{\nabla \phi}{|\nabla \phi|} \cdot \nabla \phi=\operatorname{sgn}\left(\phi^{0}\right),
$$

where $\phi^{0}$ is the original value of the level-set function which defines the interface and $\phi$ is the updated level-set function. If this equation is solved to the steady state, the entire domain of interest is reinitialized to be a signed distance function. We solve equation (22) with an upwind approach similar to that in [23]. In our work reinitialization was performed every 100 iterations or when the gradient differed greatly from the value of unity. Results did not change if reinitialization was performed more often.

To simulate the cracking of materials it is necessary to have an initial level-set function which describes the initial state of the system. This is accomplished by first locating the initial crack interface on the numerical grids. If the crack interface passes through a grid point the initial value of that grid point is set to zero. For grid points directly next to the interface the distance to the interface is calculated and used as the initial level-set value, with the sign depending on whether the grid point is inside or outside the crack. Then regions inside the crack are all assigned the value of -1 while regions outside the crack are assigned the value of 1 . We then apply the reinitialization procedure to replace this crude level set with a smooth signed distance function, which serves as the starting level set for the simulation.

The following outlines the entire procedure of simulation. After the initial level set is defined, the elastic field can be calculated by iterating equation (17). In a band of grid points directly next to the interface the interfacial curvature is calculated by equation (3). The source term $S(\phi)$ is also calculated in this band. Apply equation (21) to extend $S$ smoothly from the interface to other regions. Then $\phi$ and $S$ are transformed into Fourier space and the level set is updated by equation (20). Inverse Fourier transform gives the updated $\phi$ in real space. The procedure repeats until a desired time. 


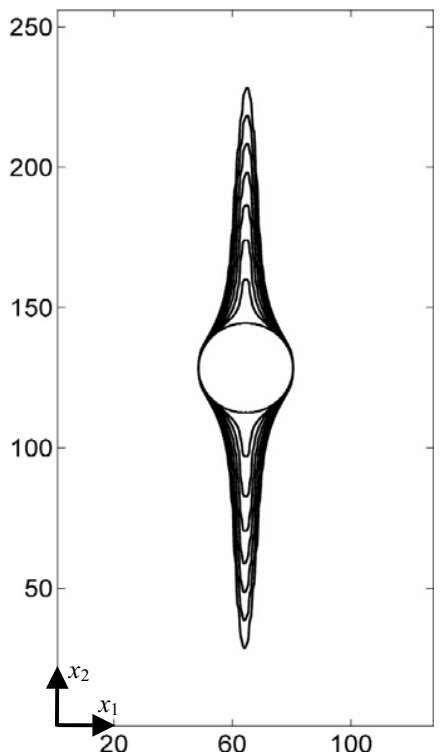

Figure 3. The evolution of a circular hole under a tensile strain of 0.045 in the $x_{1}$ direction. The zero contours at different time steps show the extension of the crack surface. The time between two neighbouring contours is $10 t_{0}$.

\section{Numerical simulations}

Representative results are presented in the following. Unless otherwise stated, simulations were run on a grid of 128 by 256 with a grid spacing of $l_{0}$. In this paper we use the elastic properties of $\mathrm{SiO}_{2}$ in all our simulations. The Young's modulus takes the value of $E=70 \mathrm{GPa}$ and Poisson's ratio is $v=0.17$ [36]. Take $l_{0}=1 \mathrm{~nm}$. The surface energy density is on the order of $1 \mathrm{~J} \mathrm{~m}^{-2}$. These values lead to $\omega_{0}=10^{9} \mathrm{~J} \mathrm{~m}^{-3}$.

First consider the evolution of an initially circular hole under a uniaxial loading in the $x_{1}$ direction, as shown in figure 3. On the surface of the hole, the two extreme points in the $x_{2}$ direction have the largest stress concentration factor of three. When the load is large enough, mode-I cracks may initiate from these high stress points. The proposed level-set approach was used to understand the morphology evolution and crack propagation process. The size of the hole is small compared to the calculation domain so that the film can be treated as infinitely large. In such a situation there is an analytical result for the elastic field. A tensile strain of 0.045 was applied in the simulation. It was verified that the elastic field distribution and stress concentration factor calculated by the numerical approach in section 3.1 agreed well with the theoretical prediction. The level set was evolved and the zero contours at different time steps, which defined the locations of the crack surface, were plotted in figure 3 . The time between two neighbouring contours is $10 t_{0}$. The hole first starts to form small cusps at regions of high stress. The increasing curvature of the cusps leads to higher stress concentration and promotes the extension of cusps into cracks. The simulation shows that over time the crack front reaches an equilibrium profile, leading to a constant propagation velocity. This can be understood in the following. When the crack is long and maintains the tip profile, the relaxed elastic energy associated with unit crack advancement is almost

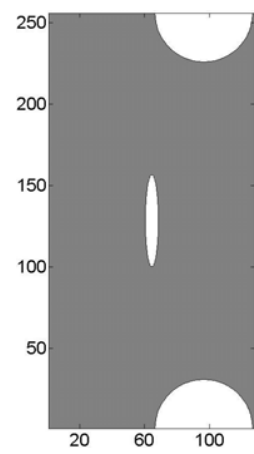

(a)

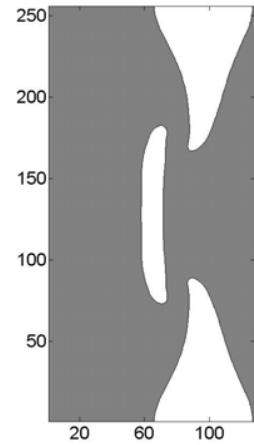

(d)

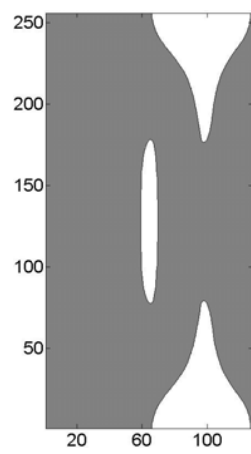

(b)

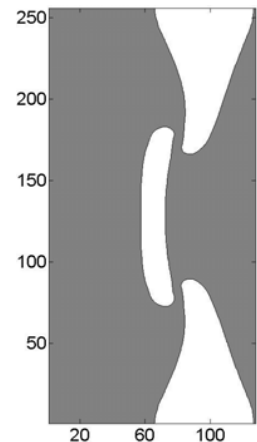

(e)

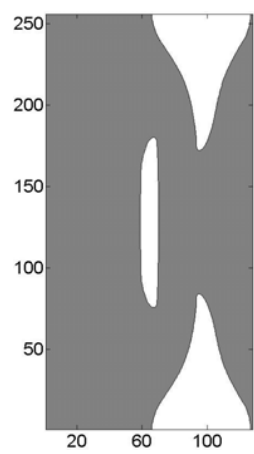

(c)

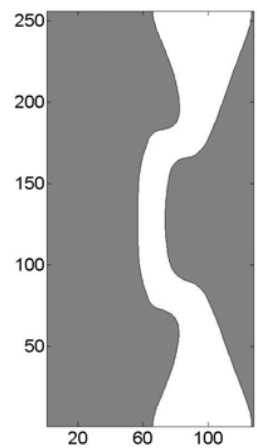

(f)
Figure 4. Cracks curve towards empty etched spaces. The tensile strains of 0.025 and 0.005 were applied in the $x_{1}$ and $x_{2}$ directions, respectively. Shown for time (a) 0 , (b) $200 t_{0}$, (c) $300 t_{0}$, (d) $400 t_{0}$, (e) $600 t_{0}$ and (f) $700 t_{0}$.

constant. The increase of the surface energy associated with the newly created surfaces is also constant for any unit crack advancement. The energy difference constitutes a fixed driving force for cracking. Thus the crack propagates at a constant velocity when the energy dissipation rate keeps invariant. The constant kinetic coefficient $D$ in equation (7) essentially implies a fixed dissipation rate during evolution. The sequence in figure 3 agrees with results from other simulations using different approaches [16, 19].

Recent experiments have shown that nanocrack patterns can be used as templates to fabricate nanowires $[6,5]$. Cracks may deviate from straight propagation paths and curve towards empty etched spaces. The observation suggests that material inhomogeneity in a film may be exploited to direct crack path and obtain desired nanocrack patterns. The interactions of two parallel cracks have been studied in mechanics literature. For instance, Xia and Hutchinson considered two parallel semi-infinite cracks [37]. The proposed model allows the investigation of arbitrary crack paths with finite crack length and size under various loading conditions. Figure 4(a) shows two etched holes at the edges and a small elliptical crack in the centre. The white colour indicates etched empty regions while the dark colour indicates the film material. Note that the boundary conditions are periodic. The two seeming semi-holes are actually complete circular holes. The tensile strains of 0.025 and 0.005 were applied in the $x_{1}$ and $x_{2}$ directions, respectively. The snapshots at various times during the evolution are shown in figures 4(b)-(f). Figure 4(b) shows that crack initiation at the edges of the holes and propagation 


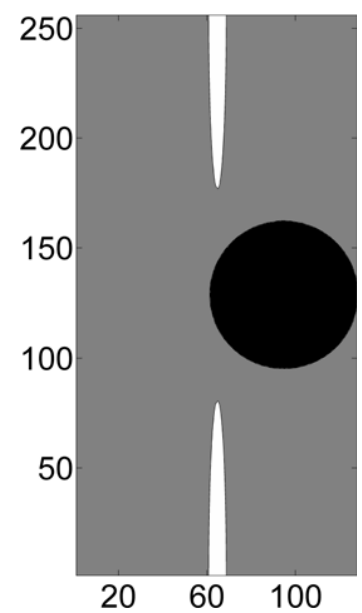

(a)

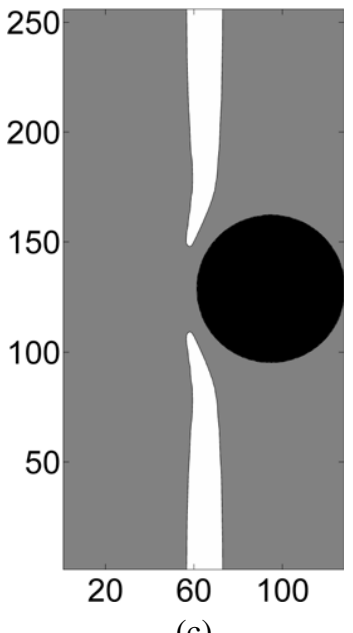

(c)
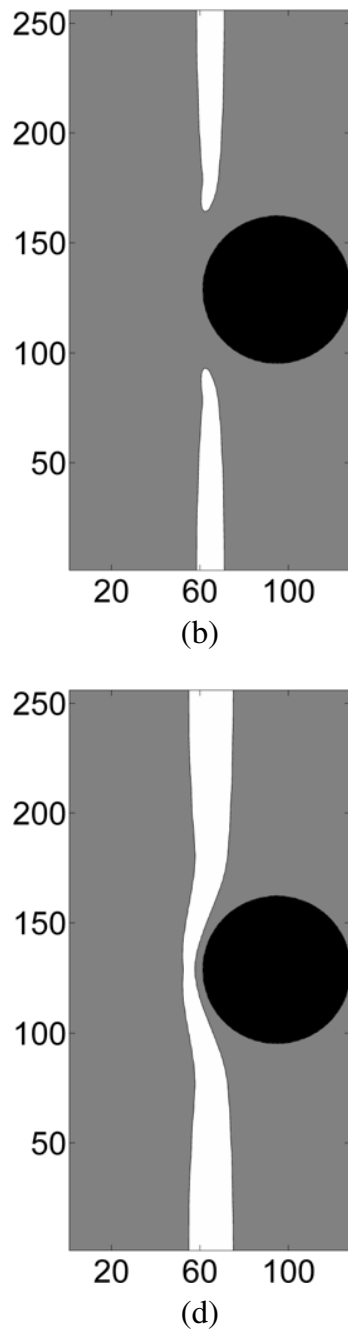

(d)

Figure 5. Deflection of crack paths by stiff materials. A biaxial tensile misfit strain of 0.025 was applied. The cracks orient themselves to parallel to the edge of the stiff region. Shown for time (a) 0 , (b) $7 t_{0}$, (c) $14 t_{0}$, (d) $22 t_{0}$.

of the elliptical crack act simultaneously. When the crack tips are close, as shown in figure 4(c), they begin to sense each other. The interaction causes the crack tips to turn and eventually merge. This crack reorientation process is captured by figures $4(\mathrm{c})-(\mathrm{f})$. The simulation suggests that by proper design crack interaction may be exploited to create nanowire interconnects between different components in an electronic system.

In contrast to etched spaces, patterning stiff materials in a homogenous film may deflect cracks. Figure 5 shows an example. In this calculation the grid spacing was taken to be $10 l_{0}$. The $\mathrm{SiO}_{2}$ film, which is represented by the grey colour, contains two elliptic cracks indicated by the white colour. The film also contains a disk of higher stiffness indicted by the black colour. For the elastic constants of the stiff material we took $C_{11}=165.7 \mathrm{GPa}, C_{12}=63.9 \mathrm{GPa}$ and $C_{44}=$ 79.6 GPa, which are typical for silicon. For comparison, with $E=70 \mathrm{GPa}$ and $v=0.17$, the film material of the grey region has $C_{11}=75.2 \mathrm{GPa}, C_{12}=15.4 \mathrm{GPa}$ and $C_{44}=29.9 \mathrm{GPa}$. A biaxial tensile misfit strain of 0.02 was applied. Figures 5(b)(d) show that the cracks orient themselves parallel to the edge

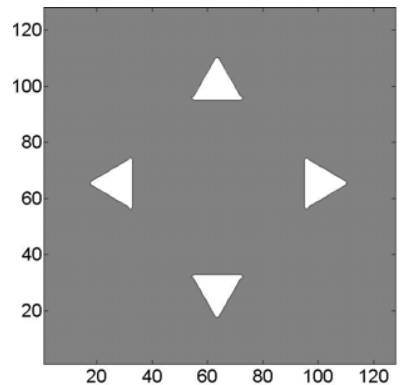

(a)

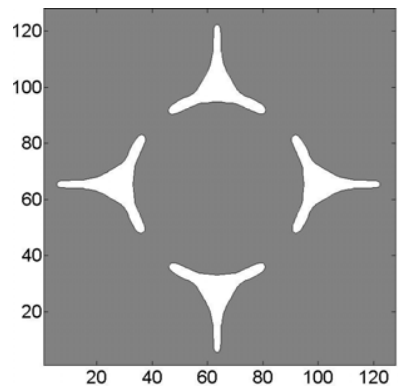

(c)

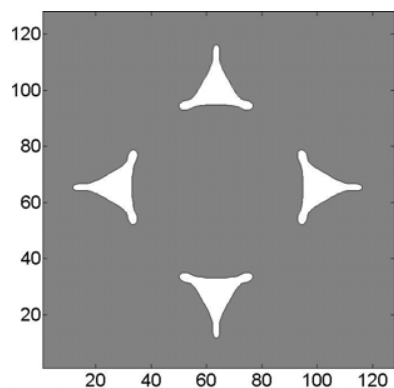

(b)

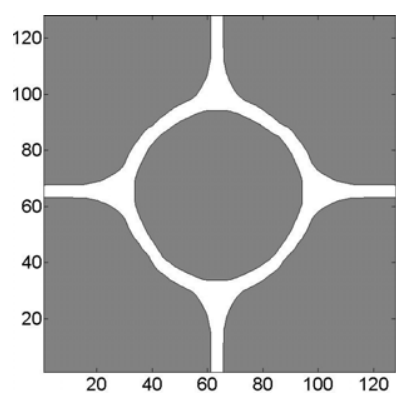

(d)
Figure 6. Application of controlled nanocrack formation allows making nanowires difficult to obtain in other methods. The film contains four etched triangles. A biaxial tensile misfit strain of 0.045 was applied. Cracks extend, reorient and eventually merge into a circular nanocrack ring. Shown for time (a) 0 , (b) $2 t_{0}$, (c) $4 t_{0}$ and (d) $8 t_{0}$.

of the stiff region. Initially the cracks propagate in a straight manner. As the cracks move closer to the stiff regions they begin to deflect. This behaviour is consistent with experimental results on graded materials $[30,31]$. It should be noted that when an interface is weak (not well bonded, defects, etc) a crack may deflect toward the interfacial region, as observed in several experiments, to relax more energy. This process is comparable to a crack deflecting toward another crack (treat the interface as a crack or void). Figure 5(d) shows that the two cracks meet and form a continuous curved channel near the stiff region.

Application of controlled nanocrack formation allows us to make nanowires which are difficult to obtain by other methods. To demonstrate the capability, consider the fabrication of nanowire rings. Figure 6(a) shows a film containing four etched triangles. The domain size is $128 \times 128$ with a grid spacing of $2 l_{0}$. A biaxial tensile misfit strain of 0.045 was applied to the film. Cracks begin to initiate at the corners of the triangles. These cracks extend, reorient and eventually merge into a circular nanocrack ring. Nanowire rings with designed location and arrangement can be produced by filling the crack and subsequently removing the $\mathrm{SiO}_{2}$ film. We found through simulations that diverse crack patterns could be produced by properly designing the etched regions.

\section{Conclusions}

Experiments have shown that directed cracking of thin films can be utilized to make nanowires. To fully explore the potential of this approach, a computational model needs to 
Design nanocrack patterns in heterogeneous films

be developed to predict the crack patterns. This paper proposes a level-set approach that is capable of determining cracking patterns in various system configurations. The approach and physical concept to model crack extension is non-traditional. Particularly, the work gives insight into nanoscale cracks where the crack tip can no longer be viewed as infinitely sharp. The study helps to understand the physical process and allows prediction of nanocrack paths in films of multiple materials. The simulations suggest that when a crack approaches an etched space the elastic interaction causes an attraction between them. A crack reorients its propagation path to encounter the etched region at a right angle. On the other hand, a region patterned with materials stiffer than the film deflects a crack tip away from it. The proposed approach allows designing etched/stiffer regions in a film and promises a significant degree of experimental control in producing predetermined crack patterns, which provides much flexibility in making various nanowires difficult to obtain with other methods.

\section{Acknowledgment}

The authors acknowledge financial support from National Science Foundation Career Award DMI-0348375.

\section{References}

[1] Hollatz M, Bobeth M, Pompe W and Marx V 1996 Orientation dependent crack patterns in alumina films on NiAl single crystals due to spherical indentation Acta Mater. 44 4149-59

[2] Weh L and Venthur A 2004 Crack patterns in thin polymer layers Macromol. Mater. Eng. 289 227-37

[3] Czyzewski J J, Krajniak J and Klein S 2004 The STM observations of the nano-crackings on a (110) TiO2 surface due to quenching of the crystal Appl. Surf. Sci. 227 144-50

[4] Tambe N S and Bhushan B 2004 In situ study of nano-cracking in multilayered magnetic tapes under monotonic and fatigue loading using an AFM Ultramicroscopy $100359-73$

[5] Alaca B E, Sehitoglu H and Saif T 2004 Guided self-assembly of metallic nanowires and channels Appl. Phys. Lett. 84 4669-71

[6] Adelung R, Aktas O C, Franc J, Biswas A, Kunz R, Elbahri M, Kanzow J, Schurmann U and Faupel F 2004 Strain-controlled growth of nanowires within thin-film cracks Nat. Mater. 3 375-9

[7] Huang Y, Duan X F, Cui Y, Lauhon L J, Kim K H and Lieber C M 2001 Logic gates and computation from assembled nanowire building blocks Science 294 1313-7

[8] Melosh N A, Boukai A, Diana F, Gerardot B, Badolato A, Petroff P M and Heath J R 2003 Ultrahigh-density nanowire lattices and circuits Science $\mathbf{3 0 0} 112-5$

[9] Kramer N, Birk H, Jorritsma J and Schonenberger C 1995 Fabrication of metallic nanowires with a scanning tunneling microscope Appl. Phys. Lett. 66 1325-7

[10] Manoharan H C, Lutz C P and Eigler D M 2000 Quantum mirages formed by coherent projection of electronic structure Nature $\mathbf{4 0 3}$ 512-5

[11] Forrer P, Schlottig F, Siegenthaler H and Textor M 2000 Electrochemical preparation and surface properties of gold nanowire arrays formed by the template technique J. Appl. Electrochem. 30 533-41
[12] Ji C X and Searson P C 2002 Fabrication of nanoporous gold nanowires Appl. Phys. Lett. 81 4437-9

[13] Zhang X Y, Zhang L D, Lei Y, Zhao L X and Mao Y Q 2001 Fabrication and characterization of highly ordered $\mathrm{Au}$ nanowire arrays J. Mater. Chem. 11 1732-4

[14] Liang J, Huang R, Prevost J H and Suo Z 2003 Evolving crack patterns in thin films with the extended finite element method Int. J. Solids Struct. 40 2343-54

[15] Hutchinson J W and Evans A G 2000 Mechanics of materials: Top-down approaches to fracture Acta Mater. 48 125-35

[16] Eastgate L O, Sethna J P, Rauscher M, Cretegny T, Chen C S and Myers C R 2002 Fracture in mode I using a conserved phase-field model Phys. Rev. E 65036117

[17] Karma A, Kessler D A and Levine H 2001 Phase-field model of mode III dynamic fracture Phys. Rev. Lett. 8704045501

[18] Marconi V I and Jagla E A 2005 Diffuse interface approach to brittle fracture Phys. Rev. E 71036110

[19] Salac D and Lu W 2006 Controlled nanocrack pattens for nanowires J. Comput. Theor. Nanosci. $3263-8$

[20] Spatschek R, Hartmann M, Brener E, Muller-Krumbhaar H and Kassner K 2006 Phase field modeling of fast crack propagation Phys. Rev. Lett. 96015502

[21] Jagla E A 2004 Maturation of crack patterns Phys. Rev. E 69056212

[22] Osher S and Fedkiw R P 2001 Level set methods: An overview and some recent results J. Comput. Phys. $169463-502$

[23] Peng D P, Merriman B, Osher S, Zhao H K and Kang M J 1999 A PDE-based fast local level set method J. Comput. Phys. 155 410-38

[24] Smereka P 2003 Semi-implicit level set methods for curvature and surface diffusion motion J. Sci. Comput. $19439-56$

[25] Stolarska M, Chopp D L, Moes N and Belytschko T 2001 Modelling crack growth by level sets in the extended finite element method Int. J. Numer. Methods Eng. 51 943-60

[26] Osher S and Sethian J A 1988 Fronts propagating with curvature-dependent speed-algorithms based on Hamilton-Jacobi formulations J. Comput. Phys. $7912-49$

[27] Sethian J A and Smereka P 2003 Level set methods for fluid interfaces Annu. Rev. Fluid Mech. 35 341-72

[28] Chen S, Merriman B, Kang M, Caflisch R E, Ratsch C, Cheng L T, Gyure M, Fedkiw R P, Anderson C and Osher S 2001 A level set method for thin film epitaxial growth J. Comput. Phys. 167 475-500

[29] Li Z L, Zhao H K and Gao H J 1999 A numerical study of electro-migration voiding by evolving level set functions on a fixed Cartesian grid J. Comput. Phys. 152 281-304

[30] Tilbrook M T, Moon R J and Hoffman M 2005 Crack propagation in graded composites Compos. Sci. Technol. 65 201-20

[31] Tilbrook M T, Rozenburg K, Steffler E D, Rutgers L and Hoffman M 2006 Crack propagation paths in layered, graded composites Composites B 37 490-8

[32] Yu H C and Lu W 2005 Dynamics of the self-assembly of nanovoids and nanobubbles in solids Acta Mater. 53 1799-807

[33] Kim D and Lu W 2006 Three-dimensional model of electrostatically induced pattern formation in thin polymer films Phys. Rev. B 73035206

[34] Chopp D L and Sethian J A 1999 Motion by intrinsic Laplacian of curvature Interfaces Free Bound. 1 1-18

[35] Russo G and Smereka P 2000 A remark on computing distance functions J. Comput. Phys. 163 51-67

[36] Kim M T 1996 Influence of substrates on the elastic reaction of films for the microindentation tests Thin Solid Films 28312

[37] Xia Z C and Hutchinson J W 2000 Crack patterns in thin films J. Mech. Phys. Solids 48 1107-31 